\title{
Differential efficacy of passive immunisation against infection by Lyme disease spirochaetes transmitted by partially fed vector ticks
}

\author{
CHIEN-MING SHIH and LI-PING LIU \\ Department of Parasitology and Tropical Medicine, National Defense Medical Center, PO Box 90048-506, \\ Taipei, Taiwan, Republic of China
}

\begin{abstract}
The efficacy of passive immunisation against tick-transmitted Lyme disease spirochaetal infection was determined in relation to the duration of previous feeding of infected vector ticks. Thus, mice challenged with spirochaete-infected unfed or partially fed nymphal ticks were passively immunised with monoclonal and polyclonal antibodies against the Lyme disease spirochaete (Borrelia burgdorferi) at various intervals after tick attachment. Spirochaetal infection in challenged mice and engorged ticks was verified by xenodiagnosis and indirect immunofluorescent antibody assay, respectively. Although tick-transmitted spirochaetal infection could be aborted by anti-OspA antibodies and hyperimmune antiserum, nearly all immunised mice challenged with infected ticks that had previous 36-h attachment became infected. More than $72 \%$ of the nymphal ticks used in this challenge retained their $B$. burgdorferi infection after engorgement on mice immunised with anti-spirochaete antibodies, and their subsequent infectivity to mice remained effective. It is concluded that a higher efficiency of transmission by partially fed infected nymphs and a lower efficacy of passive immunisation against infection result from an effect of previous feeding of infected ticks that activates antigenic change and enables the spirochaetes to circumvent OspA-based humoral immunity.
\end{abstract}

\section{Introduction}

Passive immunisation appears to protect experimental animals from infection and disease caused by the aetiological agent of Lyme disease, Borrelia burgdorferi [1-6]. Passive administration of the antibodies produced against the major outer surface proteins (Osps) of B. burgdorferi may prevent spirochaetal infection and the subsequent development of disease $[2,4,6]$. Similarly, active immunisation with Osps of spirochaetes to elicit borreliacidal and protective antibodies also prevents subsequent persistent infection in hosts challenged by either bites of infected ticks or cultured spirochaetes [4,7-14]. These observations demonstrate that humoral immunity protects hosts against infection when challenged by the agent of Lyme disease.

Efficiency of transmission of Lyme disease spirochaetes correlates directly with the duration of attachment of the infective ticks $[15,16]$. Spirochaetal

Received 6 Nov. 1997; revised version accepted 5 Jan. 1998. Corresponding author: Dr C-M. Shih. infection can be transmitted efficiently by unfed (flat) infected nymphal ticks within $48 \mathrm{~h}$ of attachment $[5,16,17]$. However, the crucial period required for partially fed infected nymphs to transmit the agent of Lyme disease is less than that required by unfed infected nymphs [17]. Although passive transfer of immune serum or monoclonal antibodies (MAbs) is sufficient for protection of mice against tick-delivered spirochaetal infection $[5,6]$, the protective immunity afforded by passive immunisation is observed within a narrow time window before the transmission of spirochaetes [5]. In addition, antigenic variation of $B$. burgdorferi during tick feeding has been reported [18], and a temperature-related differential expression of Osps has also been demonstrated [19]. For these reasons, the efficacy of passive immunisation in preventing spirochaetal infection transmitted by partially fed infected nymphs needs to be further defined.

Spirochaetal infection may be transmitted more efficiently to the host by partially fed nymphal ticks and the duration of previous feeding of infected nymphal ticks is an alternative factor that may influence the efficacy of passive immunisation in protecting mice against Borrelia infection. To explore 
this possibility, laboratory-reared infected nymphs were permitted to pre-feed on spirochaete-free mice for various periods after tick attachment and then allowed to re-attach on passively immunised mice until repletion, and the efficacy of protective immunity in mice against spirochaetal challenge by these partially fed infected nymphs was compared. In particular, the ability of anti-spirochaete antibodies to eliminate spirochaetes within engorged nymphs was defined, as well as their effect on subsequent infectivity for the host.

\section{Materials and methods}

\section{Tick colony}

The uninfected larval vector ticks used in this study were the third-generation progeny of adult Ixodes dammini (also known as I. scapularis) originally collected from the Crane Wildlife Reservation (Ipswich, MA, USA). This colony was introduced to our laboratory in November 1994 and was maintained free of inherited spirochaetal infection as described previously [20]. Spirochaete-infected nymphs were derived from the engorged larvae that had fed on spirochaeteinfected mice. All ticks were stored in mesh-covered plaster-bottomed plastic vials at $22^{\circ} \mathrm{C}, 95 \%$ relative humidity, and $16 \mathrm{~h}$ of light per day.

\section{Experimental animals}

Groups of 4-5-week-old spirochaete-free mice $(\mathrm{C} 3 \mathrm{H} / \mathrm{HeNCrj})$ were purchased from the animal supply centre of National ChengKung University Medical Center (Tainan, Taiwan). All mice were ear tagged and caged by group according to the type of antibody to be infused. All experiments were conducted under the Guidelines for Animal Experiments of the National Science Council, Taiwan, ROC.

\section{Spirochaete strain}

The spirochaetal isolate (JD1) of B. burgdorferi sensu stricto used in the present study was originally derived from naturally infected vector ticks swept from vegetation at the endemic site of the Crane Wildlife Reservation as described previously [21]. This isolate reacted with MAb $\mathrm{H} 5332$ [22] and B. burgdorferispecific DNA probes [23], and it is highly infectious to rodent hosts [24-26]. It had been introduced into this laboratory in November 1994 and was maintained in a system of alternate passage between vector ticks and laboratory mice, as described previously [20].

\section{Tick attachment and feeding}

To permit successful attachment, spirochaete-free mice $(\mathrm{C} 3 \mathrm{H} / \mathrm{HeNCrj})$ were anaesthetised by injection of $0.2 \mathrm{ml}$ pentobarbital solution $(0.1 \%)$, shaved with an animal clipper and restrained individually in a small wire cage, as described previously [17]. Infected nymphal vector ticks ( 35 for each mouse) were randomly placed on these mice and allowed to attach for specified periods. Because ticks may take from a few minutes to an hour to attach, the duration of attachment was measured beginning at $1 \mathrm{~h}$ after exposure to mice. Ticks that failed to attach within $1 \mathrm{~h}$ were removed and discarded. Attached nymphal ticks were removed at various intervals $(24,36$ and $48 \mathrm{~h}$ ) after attachment by gentle traction with fine forceps. Any tick that was injured during removal was discarded. These partially fed infected nymphs were subsequently allowed to re-attach on passively immunised mice until fully engorged. The engorged nymphs were collected and stored in separate mesh-covered vials for further investigations of the survival of spirochaetes within the engorged ticks and their subsequent spirochaetal infectivity for non-infected mice.

\section{Antiserum and MAbs}

Hyperimmune polyvalent rabbit serum was produced by injecting cultured JD1 strain spirochaetes into New Zealand White rabbits and the antiserum titre $(>2048)$ was determined by the indirect immunofluorescent antibody test, as described previously [20]. Anti-OspA activity was dominantly detected in the hyperimmune serum. MAbs against the OspA (H5332) or OspB (H6831) of B. burgdorferi (courtesy of Dr A. G. Barbour, University of Texas, USA) were derived from the undiluted hybridoma supernates. For comparison, sera collected from non-infected rabbit or mouse were used as the control regimen.

\section{Passive immunisation and challenge of mice}

To determine the efficacy of passive immunisation against tick-delivered spirochaetal infection, six mice from each group were passively immunised with $0.5 \mathrm{ml}$ of undiluted hyperimmune rabbit serum or MAbs against $B$. burgdorferi by intraperitoneal infusion at various intervals (1, 3 and 5 days) after attachment of unfed (flat) infected nymphal ticks. For comparison, mice challenged with flat infected nymphal ticks were infused with normal rabbit or mouse serum at 1 and 3 days after attachment. Similarly, mice challenged with partially fed infected nymphal ticks were also passively immunised with antiserum or MAbs against $B$. burgdorferi at intervals (1, 2 and 3 days) after reattachment of partially fed infected nymphal ticks. In general, each mouse was challenged with 3-5 unfed or partially fed infected nymphal ticks until fully engorged.

\section{Tick homogenates and infectivity assay}

Homogenates of engorged nymphs that had previously fed for various intervals $(24,36$ and $48 \mathrm{~h})$ and subsequently re-attached on passively immunised mice 
until repletion were prepared by grinding 5-7 engorged nymphs with phosphate-buffered saline (PBS) in a 1-ml Dounce tissue grinder (Wheaton Instruments, Millville, NJ, USA). Spirochaetal infectivity for mice was tested by inoculating mice with $0.3 \mathrm{ml}$ of tick homogenate intradermally at multiple sites, as described previously [25] and the spirochaetal infection in inoculated mice was determined by xenodiagnosis 1 month later.

\section{Detection of spirochaetes in engorged ticks}

To determine the ability of passive immunisation to destroy spirochaetes within the engorged nymphs, manually removed infected nymphal ticks were allowed to re-attach and engorge on passively immunised mice until repletion. All engorged nymphs were dissected 2 weeks later and examined for evidence of spirochaetes by an indirect immunofluorescent antibody assay, as described previously [26]. Briefly, the midgut and other internal tick organs were dissected into a drop of PBS ( $\mathrm{pH}$ 7.2) on ringed slides, squashed with a coverslip, and permitted to dry. Preparations were fixed in acetone for $10 \mathrm{~min}$, treated with high titre polyvalent rabbit anti-spirochaete antiserum, and then stained with a fluorescein isothiocyanate (FITC)-conjugated goat antirabbit immunoglobulin G (F0382, Sigma). Slides were washed in PBS, air-dried, mounted with buffered glycerol, overlaid with a coverslip and examined for spirochaetes by epifluorescence microscopy.

\section{Determination of infection in mice}

A xenodiagnostic procedure was used to determine whether passively immunised mice became persistently infected after challenge with either unfed infected nymphs or partially fed infected nymphs. Briefly, challenged mice of each group were restrained individually in a small wire cage, and laboratory-reared non-infected larval ticks ( $60-80$ for each mouse) were placed randomly on these mice until repletion. At least 20 engorged larvae from each mouse were dissected 2 weeks later, and all ticks were examined for spirochaetal infection by an indirect immunofluorescent antibody (IFA) assay. Serological evidence of infection was also confirmed by seroconversion with an increase in IFA titre to $B$. burgdorferi.

\section{Results}

\section{Abortion of tick-transmitted spirochaetal infection} by passive immunisation

A preliminary experiment determined whether the tickdelivered spirochaetal infection could be aborted by passive immunisation with various antibodies produced against the Lyme disease spirochaetes. Thus, mice challenged with spirochaete-infected unfed nymphal ticks were immunised with hyperimmune rabbit antiserum or MAbs against the Osps (OspA or OspB) of $B$. burgdorferi at intervals of 1,3 and 5 days after attachment of unfed infected ticks. For comparison, mice infused with normal rabbit or mouse serum were also challenged with unfed infected nymphs. After xenodiagnosis, no spirochaetal infection was detected in any mouse immunised with polyvalent antiserum or MAbs against OspA at 1 day after unfed infected nymphs had attached. Only one mouse immunised with MAbs against OspB was protected from infection by tick challenge (Table 1). Generally, mice became infected mainly when they were passively immunised with antiserum or MAbs 3 days after unfed infected ticks had attached. In contrast, all mice became infected when they were infused with normal rabbit or mouse serum at 1 day after the unfed infected ticks had attached. These results demonstrate that ticktransmitted spirochaetal infection can be aborted by

Table 1. Efficacy of passive immunisation of mice against Lyme disease infection after challenge by unfed infected ticks

\begin{tabular}{lccc}
\hline $\begin{array}{l}\text { Type of } \\
\text { antibody } \\
\text { infused* }\end{array}$ & $\begin{array}{c}\text { Days tick } \\
\text { attached } \\
\text { until infused }\end{array}$ & Number tested & Infected (\%) \\
\cline { 3 - 4 } Anti-OspA & 1 & 6 & 0 \\
H5332 & 3 & 6 & 67 \\
Anti-OspB & 5 & 6 & 100 \\
H6831 & 1 & 6 & 83 \\
& 3 & 6 & 100 \\
Polyvalent & 5 & 6 & 100 \\
JD1 & 1 & 6 & 0 \\
& 3 & 6 & 67 \\
Rabbit sera & 5 & 6 & 100 \\
& 1 & 3 & 100 \\
Mouse sera & 3 & 3 & 100 \\
& 1 & 3 & 100 \\
& 3 & 3 & 100 \\
\hline
\end{tabular}

*Undiluted hybridoma supernates (anti-OspA or anti-OspB), polyvalent rabbit anti-spirochaete (JD1) serum and normal rabbit or mouse serum were infused intraperitoneally in mice. 
passive immunisation only when antibody is administered $24-48 \mathrm{~h}$ after unfed infected ticks had attached. This probably corresponds to a period prior to the deposition of Lyme disease spirochaetes by vector ticks.

\section{Efficacy of passive immunisation against} spirochaetal infection challenged by partially fed infected ticks

To determine the efficacy of passive immunisation in the protection of mice against spirochaetal infection transmitted by the partially fed infected nymphal ticks, spirochaete-infected nymphal ticks were removed from attached hosts at intervals of 24, 36 and $48 \mathrm{~h}$ after initial attachment and used to challenge the mice immunised with various anti-spirochaete antibodies at intervals of 1,2 and 3 days after re-attachment of partially fed infected nymphs. Spirochaetal infection was detected in the majority $(>67 \%)$ of mice immunised with hyperimmune antiserum or MAbs against OspA at 1 day after partially fed infected nymphs had reattached (Table 2). No mice were protected from spirochaetal infection when they were infused with MAbs against OspB. Regardless of the protective antibodies infused into mice, virtually all mice became infected when they were challenged with infected nymphal ticks that had previously fed on hosts $>36 \mathrm{~h}$ after initial attachment. These results indicate that spirochaetal infection is transmitted more efficiently by partially fed infected ticks and passive immunisation did not protect mice against spirochaetal infection from challenge by partially fed infected nymphal ticks.

Elimination of spirochaetes within infected ticks by passively infused anti-spirochaete antibodies

The ability of passively infused anti-spirochaete antibodies to eliminate spirochaetes resident in the infected nymphal ticks that had previously fed on hosts $>24 \mathrm{~h}$ after initial attachment, and subsequently engorged on passively immunised mice until repletion was then assessed. Two weeks after repletion, spirochaetes were detected within the majority $(>72 \%$ ) of engorged ticks that had fed on mice infused with polyvalent antiserum or MAbs against B. burgdorferi (Table 3). In contrast, almost all ticks that had engorged on mice infused with normal serum remained infected. These observations indicate that spirochaetes resident in the partially fed infected ticks were not eliminated by passively infused antibodies.

\section{Infectivity to hosts by spirochaetes that survive} within ticks

The study determined whether spirochaetes survived within the engorged ticks that had fed on passively

Table 2. Efficacy of passive immunisation of mice against Lyme disease infection after challenge by partially fed infected ticks

\begin{tabular}{|c|c|c|c|c|}
\hline \multirow{2}{*}{$\begin{array}{l}\text { Duration (h) } \\
\text { of previous } \\
\text { attachment of } \\
\text { infected ticks }\end{array}$} & \multirow{2}{*}{$\begin{array}{l}\text { Type of } \\
\text { antibody } \\
\text { infused* }\end{array}$} & \multirow{2}{*}{$\begin{array}{l}\text { Days tick } \\
\text { re-attachment } \\
\text { until infused }\end{array}$} & \multicolumn{2}{|c|}{ Infection in mice } \\
\hline & & & $\begin{array}{l}\text { Number } \\
\text { tested }\end{array}$ & $\begin{array}{c}\text { Infected } \\
(\%)\end{array}$ \\
\hline \multirow[t]{9}{*}{24} & Anti-OspA & 1 & 6 & 67 \\
\hline & & 2 & 6 & 83 \\
\hline & & 3 & 6 & 100 \\
\hline & Anti-OspB & 1 & 6 & 100 \\
\hline & & 2 & 6 & 100 \\
\hline & & 3 & 6 & 100 \\
\hline & Polyvalent & 1 & 6 & 67 \\
\hline & & 2 & 6 & 83 \\
\hline & & 3 & 6 & 100 \\
\hline \multirow[t]{9}{*}{36} & Anti-OspA & 1 & 6 & 83 \\
\hline & & 2 & 6 & 100 \\
\hline & & 3 & 6 & 100 \\
\hline & Anti-OspB & 1 & 6 & 100 \\
\hline & & 2 & 6 & 100 \\
\hline & & 3 & 6 & 100 \\
\hline & Polyvalent & 1 & 6 & 83 \\
\hline & & 2 & 6 & 100 \\
\hline & & 3 & 6 & 100 \\
\hline \multirow[t]{9}{*}{48} & Anti-OspA & 1 & 6 & 100 \\
\hline & & 2 & 6 & 100 \\
\hline & & 3 & 6 & 100 \\
\hline & Anti-OspB & 1 & 6 & 100 \\
\hline & & 2 & 6 & 100 \\
\hline & & 3 & 6 & 100 \\
\hline & Polyvalent & 1 & 6 & 100 \\
\hline & & 2 & 6 & 100 \\
\hline & & 3 & 6 & 100 \\
\hline
\end{tabular}

${ }^{*} \mathrm{MAb}$ to OspA (H5332) or OspB (H6831) and polyvalent rabbit antiserum (JD1) were infused in mice. 
Table 3. Elimination of spirochaetes from the infected ticks that were detached while feeding and subsequently engorged on mice immunised with various anti-spirochaete antibodies

\begin{tabular}{lcc}
\hline \multirow{2}{*}{$\begin{array}{l}\text { Type of } \\
\text { antibody infused } \\
\text { in mice }\end{array}$} & \multicolumn{2}{c}{ Detection of spirochaetes in engorged ticks* } \\
\cline { 2 - 3 } & Number examined & Infected (\%) \\
\hline Anti-OspA & 42 & 83 \\
Anti-OspB & 38 & 89 \\
Polyvalent & 46 & 72 \\
Control $^{\dagger}$ & 24 & 92 \\
\hline
\end{tabular}

* Spirochaetal infection was examined 2 weeks after repletion of engorged ticks and verified by indirect immunofluorescence with polyvalent rabbit anti-spirochaete antisera as primary antibody.

Normal rabbit or mouse serum was infused in the control groups.

immunised mice until repletion remained infective to the host. Thus, tick homogenates of 5-7 engorged infected nymphs that had fed on passively immunised mice were prepared 2 weeks after engorgement and then injected into non-infected mice by intradermal inoculation. After xenodiagnosis, only one mouse remained without infection when it was inoculated with homogenate of engorged ticks that had previously fed on the host for $24 \mathrm{~h}$ after attachment and subsequently engorged on mice immunised with polyvalent antiserum or MAbs against OspA of B. burgdorferi (Table 4). Virtually all mice became infected when they were inoculated with homogenates of engorged ticks that had previously fed on hosts $>36 \mathrm{~h}$ after initial attachment. These results indicate that spirochaetal infectivity for the host was not affected by the passively infused antispirochaete antibodies.

\section{Discussion}

The effectiveness of humoral immunity against spirochaetal infection may depend on the mode of inoculation of spirochaetes and the timing of passive immunisation. Indeed, the effectiveness of passive immunisation against spirochaetal infection delivered by naturally infected ticks differs markedly from that induced by syringe inoculation, and the efficacy of passive immunisation against tick-delivered spirochae- tal infection has been attributed to the duration of attachment of unfed infected nymphal ticks $[5,6]$. In the present study, spirochaetal infection delivered by unfed infected nymphs could be aborted by passively transferred anti-spirochaete antibody administered 24$48 \mathrm{~h}$ after attachment of unfed infected nymphs. Furthermore, the transmission of Lyme disease spirochaetes by an unfed infected nymphal tick occurs mainly at $48 \mathrm{~h}$ after initial attachment of unfed infected tick $[5,16,17]$. Thus, this study confirms that passive humoral immunity against tick-transmitted spirochaetal infection is only effective if applied within a defined time period $(24-48 \mathrm{~h})$ before the deposition of spirochaetes by an unfed infected nymphal tick.

The efficacy of passive immunisation against spirochaetal infection delivered by partially fed infected nymphal ticks has never been defined. The results of the present study indicate that spirochaetal infection can be efficiently transmitted to passively immunised mice when they were challenged with partially fed infected nymphal ticks that had previously fed on the host $>24 \mathrm{~h}$ after initial attachment. These results imply that a partially fed infected nymphal tick transmits spirochaetes more rapidly than an unfed infected nymphal tick, and the span of spirochaetal transmission may be accelerated by the active dispersal of spirochaetes to tick salivary glands during the previous attachment of infected nymphal ticks [27]. Indeed, a previous study also demonstrated that partially fed infected nymphs become infectious to other hosts within only 1 day after re-attachment [17]. This is far more efficient than an unfed infected nymph. Thus, passive immunisation is not sufficient for protection of mice against spirochaetal infection challenged by partially fed infected nymphs, and the factors that modulate the effectiveness of humoral immunity against tick-transmitted spirochaetal infection remain to be defined.

The ability of passively transferred antibody to eliminate spirochaetes within the partially fed infected nymphs remains unclear. Although it had been proposed that the site of anti-spirochaete activity by

Table 4. Infectivity for mice of spirochaetes surviving within engorged ticks previously fed on a host for various periods and subsequently engorged on passively immunised mice until repletion

\begin{tabular}{|c|c|c|c|}
\hline \multirow{2}{*}{$\begin{array}{l}\text { Type of } \\
\text { antibody infused } \\
\text { in mice }\end{array}$} & \multirow{2}{*}{$\begin{array}{l}\text { Duration (h) of } \\
\text { previous feeding of } \\
\text { engorged ticks }\end{array}$} & \multicolumn{2}{|c|}{ Infectivity to mice* } \\
\hline & & Number tested & Infected $(\%)$ \\
\hline \multirow[t]{3}{*}{ Anti-OspA } & 24 & 6 & 83 \\
\hline & 36 & 6 & 100 \\
\hline & 48 & 6 & 100 \\
\hline \multirow[t]{3}{*}{ Anti-OspB } & 24 & 6 & 100 \\
\hline & 36 & 6 & 100 \\
\hline & 48 & 6 & 100 \\
\hline \multirow[t]{3}{*}{ Polyvalent } & 24 & 6 & 83 \\
\hline & 36 & 6 & 100 \\
\hline & 48 & 6 & 100 \\
\hline
\end{tabular}

* Spirochaetal infection was examined by xenodiagnosis 1 month after intradermal inoculation with $0.3 \mathrm{ml}$ of tick homogenates of engorged nymphal ticks. 
passively infused antibody was mainly within the guts of the attached ticks $[5,11]$, the present observations demonstrated that spirochaetes could be detected in the majority of the nymphal ticks that had been partially fed and subsequently engorged on passively immunised mice. Humoral immunity seemed to have no significant effect on the destruction of spirochaetes within those nymphal ticks that had previously fed on the host $>24 \mathrm{~h}$ after initial attachment. Accordingly, it is suggested that the process of previous feeding of infected nymphal ticks may actually enhance the survival of spirochaetes resident in the gut of engorged infected nymphs.

It is interesting that spirochaetes within the partially fed nymphal ticks were not vulnerable to passively immunised MAbs against the Osps of B. burgdorferi when the challenged nymphs had previously fed on a host $>24 \mathrm{~h}$ after initial attachment. Although OspA is an abundant protein on $B$. burgdorferi and vaccination based on OspA protects hosts against spirochaetal infection $[7,8,11,14]$, the expression of OspA within attached ticks may vary with the duration of feeding of the attached ticks. Indeed, previous studies demonstrated that Lyme disease spirochaetes are able to alter their outer surface during tick feeding [18], and the differential expression of OspA and OspC on $B$. burgdorferi occurs $c .24 \mathrm{~h}$ after tick attachment [6]. In addition, an OspC antibody-mediated immunity has proved to be effective in resolving established chronic Lyme disease [28] and in protection of mice against borrelia infection [29]. In the present study, spirochaetes within the partially fed nymphal ticks were not eliminated by passively infused anti-OspA MAbs and they remained infectious to other hosts. Thus, it is suggested that the previous feeding of infected nymphal ticks may actually activate the migration and antigenic change of spirochaetes within the partially fed infected nymphal ticks, and differential expression of OspC by spirochaetes may account for the circumvention of OspA-mediated humoral immunity.

Failure of vaccination against spirochaetal infection may result in exposure to partially fed infected ticks. Vector ticks feeding on infected hosts may detach prematurely because of grooming or host-derived antitick immunity [30,31], and these partially fed ticks may already be infectious to hosts and avidly seek other hosts. Indeed, re-attachment by partially fed subadult ticks occurs commonly [17,32-34]. Although the natural frequency of such partially fed infected ticks has not been determined, they may present a particularly great risk of transmission of spirochaetes.

In summary, these observations show that spirochaetal infection is transmitted more efficiently by partially fed infected nymphal ticks, and passively infused antibodies are not effective against spirochaetal infection from challenge by partially fed infected nymphs. However, the mechanisms that regulate the anti-spirochaete activity within engorged ticks remain to be determined.

Grant support from the National Science Council (NSC83-0412-B016-073) and National Defense Medical Center, Taipei, Taiwan, is acknowledged.

\section{References}

1. Johnson RC, Kodner C, Russell M. Passive immunization of hamsters against experimental infection with the Lyme disease spirochaete. Infect Immun 1986; 53: 713-714.

2. Schaible UE, Kramer MD, Eichmann K, Modolell M, Museteanu C, Simon MM. Monoclonal antibodies specific for the outer surface protein A (OspA) of Borrelia burgdorferi prevent Lyme borreliosis in severe combined immunodeficiency (scid) mice. Proc Natl Acad Sci USA 1990; 87: 3768-3772.

3. Schmitz JL, Schell RF, Lovrich SD, Callister SM, Coe JE. Characterization of the protective antibody response to Borrelia burgdorferi in experimentally infected LSH hamsters. Infect Immun 1991; 59: 1916-1921.

4. Fikrig E, Telford SR, Wallich $\mathrm{R}$ et al. Vaccination against Lyme disease caused by diverse Borrelia burgdorferi. J Exp Med 1995; 181: 215-221.

5. Shih C-M, Spielman A, Telford SR. Mode of action of protective immunity to Lyme disease spirochetes. Am J Trop Med Hyg 1995; 52: 72-74.

6. de Silva AM, Telford SR, Brunet LR, Barthold SW, Fikrig E. Borrelia burgdorferi OspA is an arthropod-specific transmission-blocking Lyme disease vaccine. J Exp Med 1996; 183: $271-275$.

7. Fikrig E, Barthold SW, Kantor FS, Flavell RA. Protection of mice against Lyme disease agent by immunizing with recombinant OspA. Science 1990; 250: 553-556.

8. Fikrig E, Barthold SW, Marcantonio N, Deponte K, Kantor FS, Flavell RA. Roles of OspA, OspB, and flagellin in protective immunity to Lyme borreliosis in laboratory mice. Infect Immun 1992; 60: $657-661$.

9. Simon MM, Schaible UE, Kramer MD et al. Recombinant outer surface protein A from Borrelia burgdorferi induces antibodies protective against spirochetal infection in mice. $J$ Infect Dis 1991; 164: 123-132.

10. Simon MM, Schaible UE, Wallich R, Kramer MD. A mouse model for Borrelia burgdorferi infection: approach to a vaccine against Lyme disease. Immunol Today 1991; 12: 11-16.

11. Fikrig E, Telford SR, Barthold SW, Kantor FS, Spielman A, Flavell RA. Elimination of Borrelia burgdorferi from vector ticks feeding on OspA-immunized mice. Proc Natl Acad Sci USA 1992; 89: 5418-5421.

12. Telford SR, Fikrig E, Barthold SW, Brunet LR, Spielman A, Flavell RA. Protection against antigenically variable Borrelia burgdorferi conferred by recombinant vaccines. $J$ Exp Med 1993; 178: $755-758$

13. Gern L, Rais O, Capiau C et al. Immunization of mice by recombinant OspA preparation and protection against Borrelia burgdorferi infection induced by Ixodes ricinus tick bites. Immunol Lett 1994; 39: 249-258.

14. Chang Y-F, Appel MJG, Jacobson RH et al. Recombinant OspA protects dogs against infection and disease caused by Borrelia burgdorferi. Infect Immun 1995; 63: 3543-3549.

15. Piesman J, Mather TN, Sinsky RJ, Spielman A. Duration of tick attachment and Borrelia burgdorferi transmission. J Clin Microbiol 1987; 25: 557-558.

16. Piesman J. Dynamics of Borrelia burgdorferi transmission by nymphal Ixodes dammini ticks. J Infect Dis 1993; 167: 1082-1085.

17. Shih C-M, Spielman A. Accelerated transmission of Lyme disease spirochetes by partially fed vector ticks. $J$ Clin Microbiol 1993; 31: 2878-2881.

18. Schwan TG, Piesman J, Golde WT, Dolan MC, Rosa PA Induction of an outer surface protein on Borrelia burgdorferi during tick feeding. Proc Natl Acad Sci USA 1995; 92: 2909-2913.

19. Stevenson B, Schwan TG, Rosa PA. Temperature-related differential expression of antigens in the Lyme disease 
spirochete, Borrelia burgdorferi. Infect Immun 1995; 63: 45354539.

20. Shih C-M, Liu L-P. Accelerated infectivity of tick-transmitted Lyme disease spirochetes to vector ticks. J Clin Microbiol 1996; 34: 2297-2299.

21. Shih C-M, Pollack RJ, Telford SR, Spielman A. Delayed dissemination of Lyme disease spirochetes from the site of deposition in the skin of mice. $J$ Infect Dis 1992; 166: 827-831.

22. Barbour AG, Tessier SL, Todd WJ. Lyme disease spirochetes and ixodid tick spirochetes share a common surface antigenic determinant defined by a monoclonal antibody. Infect Immun 1983; 41: 795-804.

23. Schwan TG, Simpson WJ, Schrumpf ME, Karstens RH. Identification of Borrelia burgdorferi and B. hermsii using DNA hybridization probes. J Clin Microbiol 1989; 27: $1734-1738$.

24. Donahue JG, Piesman J, Spielman A. Reservoir competence of white-footed mice for Lyme disease spirochetes. Am J Trop Med Hyg 1987; 36: 92-96.

25. Piesman J. Standard system for infecting ticks (Acari: Ixodidae) with the Lyme disease spirochete, Borrelia burgdorferi. J Med Entomol 1993; 30: 199--203.

26. Shih C-M, Liu L-P, Spielman A. Differential spirochetal infectivities to vector ticks of mice chronically infected by the agent of Lyme disease. $J$ Clin Microbiol 1995; 33: $3164-3168$.
27. Piesman J. Dispersal of the Lyme disease spirochete Borrelia burgdorferi to salivary glands of feeding nymphal Ixodes scapularis (Acari: Ixodidae). J Med Entomol 1995; 32: 519-521.

28. Zhong W, Stehle T, Museteanu C et al. Therapeutic passive vaccination against chronic Lyme disease in mice. Proc Natl Acad Sci USA 1997; 94: 12533-12538.

29. Bockenstedt LK, Hodzic E, Feng S et al. Borrelia burgdorferi strain-specific OspC-mediated immunity in mice. Infect Immun 1997; 65: 4661-4667.

30. Brossard M, Fivaz V. Lxodes ricinus L: mast cells, basophils and eosinophils in the sequence of cellular events in the skin of infested or re-infested rabbits. Parasitology 1982; 85: 583-592.

31. Brown SJ, Askenase PW. Rejection of ticks from guinea pigs by anti-hapten-antibody-mediated degranulation of basophils at cutaneous basophil hypersensitivity sites: role of mediators other than histamine. J Immunol 1985; 134: 1160-1165.

32. Gregson JD. Observations on the movement of fluids in the vicinity of the mouthparts of naturally feeding Dermacentor andersoni Stiles. Parasitology 1967; 57: 1-8.

33. Balashov YS. Bloodsucking ticks (Ixodoida) - vectors of diseases of man and animals. Misc Publ Entomol Soc Am 1972; 8: 301-305.

34. Piesman J. Experimental acquisition of the Lyme disease spirochete, Borrelia burgdorferi, by larval Ixodes dammini (Acari: Ixodidae) during partial blood meals. $J$ Med Entomol 1991; 28: $259-262$. 